\title{
Simvastatin in an experimental spinal cord injury model: a histopathological and biochemical evidence based study
}

\author{
Tascioglu $\mathrm{T}^{1}$, Karatay $\mathrm{M}^{2}$, Erdem $\mathrm{Y}^{1}$, Tekiner $\mathrm{A}^{1}$, Celik $\mathrm{H}^{1}$, Sahin $\mathrm{O}^{3}$, Sertbas $\mathrm{I}^{2}$, Bayar MA ${ }^{1}$ \\ Department of Neurosurgery, Ankara Training and Research Hospital, Ankara, Turkey. \\ tuncertascioglu@gmail.com
}

\begin{abstract}
AIM: In this study we tried to determine the possible neuroprotective effects of simvastatin in a rat model of Spinal Cord Injury (SCI) with the help of biochemical and histopathological tests.

METHODS: Rats were divided into 5 groups:1) SCI control, 2) Sham operated, 3) SCl with $10 \mathrm{mg} / \mathrm{kg}$ intraperitoneal simvastatin, 4) SCl with $10 \mathrm{mg} / \mathrm{kg}$ oral simvastatin, 5) SCl with $10 \mathrm{mg} / \mathrm{kg}$ subcutaneous simvastatin. After the treatment period, all rats were sacrificed; their blood and spinal cord samples were taken for biochemical and histopathological assessment.

RESULTS: When the groups were compared in terms of oedema and inflammation status, the scores of groups receiving simvastatin were better than the control and sham groups $(p=0.001$ and $p=0.038$ respectively). When the 3 treatment groups (oral, intraperitoneal and subcutaneous simvastatin groups) were compared with each other in terms of inflammation, haemorrhage and oedema, there were no significant differences between groups ( $p=0.112, p=0.797$ and $p=0.188$, respectively). NSE and S100B levels were significantly lower in the treatment groups compared to the sham group $(p=0.039$ and $p=0.004$ respectively).

CONCLUSION: According to our biochemical and histopathological findings, simvastatin $10 \mathrm{mg} / \mathrm{kg}$ has a positive impact in the spinal cord injury model in rats, regardless of route of application (Tab. 1, Fig. 5, Ref. 26). Text in PDF www.elis.sk

KEY WORDS: spinal cord injury, simvastatin, neuroprotective effect.
\end{abstract}

\section{Introduction}

Spinal cord injury (SCI) is a major cause of human morbidity, because neurons have a very limited ability for reparation. It causes devastating motor, sensory and autonomic dysfunction. The prevalence of SCI is estimated to be 250000 persons per year in the United States. Karacan et al. reported that it is 2.5 times more frequent in males than in females, and the cervical region is the most frequently effected part of spinal cord (1). Treatment options for such patients are extremely limited, high-dose methylprednisolone is the only drug approved for the medical treatment of this condition (2). Secondary injury is an important contributor to the pathological progress of SCI and is induced by several factors, such as: inflammation, oxidative stress, apoptosis and autophagy, the latest studies in this field investigated these pathways in attempts to reduce injury progression (3). Anti-inflammatory and neuroprotective treatments have also attracted interest (4), and various

${ }^{1}$ Department of Neurosurgery, Ankara Training and Research Hospital, Ankara, Turkey, ${ }^{2}$ Department of Neurosurgery, Istanbul Yeniyüzyil Univercity Medical of School, Istanbul, Turkey and ${ }^{3}$ Department of Neurosurgery, Gölbasi Sehit Ahmet Özsoy State Hospital, Ankara, Turkey

Address for correspondence: T. Tascioglu, Saglik Bakanligi Ankara Egitim ve Arastirma Hastanesi Sakarya Mh. Ulucanlar Cd. No: 89 Altindag, Ankara, Posta Kodu: 06230, Turkey.

Fax: +3124840322 experimental and clinical studies reported neuroprotective effects of compounds known to have anti-inflammatory properties (5).

Statins were introduced as HMG-CoA reductase inhibitors, which decreased cholesterol production; however, their neuroprotective and anti-inflammatory properties were evaluated extensively in various neurological disease models (6). Many intermediate products, known as piroids, are produced during cholesterol biosynthesis, some of them are; isopentyl pyrophosphate, geranyl pyrophosphate, 3,3-dimethyl allyl pyrophosphate, farnesyl pyrophosphate. These intermediates of cholesterol production reportedly induced adhesion molecules and leukocyte activation, thus leading to an activation of inflammation. When Statins inhibit cholesterol production, they also limit the bioavailability of these intermediates, which is presumably the cause of their anti-inflammatory properties (7). Furthermore, cytokines (such as: Interleukin (IL) $1 \beta, T N F \alpha$, and IL-6), which can be produced by neurons, endothelial cells and glial cells, regulate the immunological and inflammatory response. Statins have also been shown to reduce the release of these cytokines from macrophages, which is another important effect (7).

In regard to specific statins, experimental studies showed that atorvastatin decreases MCP (monocyte chemotactic protein), IL-2, IL-12 and interferon gamma secretion and showed anti-inflammatory effects on SCI (8). Simvastatin, which is a relatively new member of the HMG-CoA reductase inhibitor family, is a lipophilic 
compound that passes the blood-brain barrier with ease; due to this property, several studies claimed that it has more powerful neuroprotective effects compared to other statins $(9,10)$.

The S100 proteins constitute a large family of calcium binding proteins. S100B is the major S100 protein in the brain. When the levels of S100B increase, inflammation and apoptosis are induced, leading to neuronal damage. In many studies, a relationship between neuronal damage and elevated concentrations of S100B, especially in ischemic neuronal conditions, has been found (11, 12) Neuron specific enolase (NSE) is a gamma $(\gamma)$ subdivision of enolase, which mostly exists in the cytoplasm of neurons (13). Some researchers in literature reported NSE elevation in neurodegenerative disorders. Cooper et al. and Anand et al. mentioned in their study that there was a positive association between an acute stroke and NSE levels $(14,15)$. Today, NSE and S100B levels are suggested to be two reliable biomarkers of neuronal injury.

In literature, there are few limited studies assessing the neuroprotective properties of simvastatin in SCI. Therefore, we attempted to identify possible neuroprotective effects of simvastatin in a rat model of SCI via evaluation of biochemical and histopathological findings.

\section{Material and methods}

\section{Animals and experimental procedures}

This study was conducted in the experimental animal studies laboratory of Ankara training and research hospital. A total of 30 adult male Sprague Dawley rats weighing between 200-220 $\mathrm{g}$ were used in the study. Animals were kept in cages and maintained under standard conditions with 12-hourlight/dark cycles at a room temperature $\left(22 \pm 2{ }^{\circ} \mathrm{C}\right)$. They were fed by standard pellet diet and had tap water ad libitum for the duration of the study. The study has been carried out in accordance with the EU Directive 2010/63/EU for animal experiments, and the protocol used in the study was approved by the Local Institutional Animal Care and Ethics Committee.

In order to obtain a deep general anaesthesia, we used $2 \mathrm{mg} /$ kg ketamine-HCL (Ketalar, Parke-Davis Eczacibasi, Istanbul) and $10 \mathrm{mg} / \mathrm{kg}$ xylazainehydrochloride (Alfazyne, Alfasan International B.V. Holland). Rats were placed on a fixation board in the prone position for fixation. After sterilizing the thoracic spine region with PVD iodine (Batticon std. solution, Adeka, Samsun), we shaved the region and sterilized again with PVD iodine. A midline incision was made dorsally from T5-T12 spinal levels (16). During this procedure, interscapular distance was taken as reference and a standard spinal cord injury (SCI) was performed as follows: After carefully exposing the thoracic vertebrae, laminectomy was performed for 1 minute via a $70 \mathrm{~g}$ closing-force aneurysm clip (Yasargil FE 721Aesculap, Istanbul, Turkey), as described previously (17). Following haemostasis, paravertebral muscles and the skin were primarily sutured with $3 / 0$ Vicryl consistently with regard to anatomical layers and rats were awakened normally in a standard room temperature.

\section{Group design}

The 30 rats were divided into 5 groups, each group consisting of 6 rats. Group 1 was the control group; after taking $1 \mathrm{cc}$ of blood, they were sacrificed without any surgical procedure. Group 2 was the sham group; in which the SCI procedure was performed, no treatment was given, and they were sacrificed after obtaining 1 cc of blood. In the group 3, after performing SCI, $10 \mathrm{mg} / \mathrm{kg}$ simvastatin was given intraperitoneally at post-op $1 \mathrm{st}, 6 \mathrm{th}$, and 24 th hours after SCI, and $1 \mathrm{cc}$ of blood was taken and rats were sacrificed. Group 4 was determined as the oral simvastatin group; after the SCI procedure, starting from the first day (at the 1st hour), a daily single dose of $10 \mathrm{mg} / \mathrm{kg}$ simvastatin was given orally for 5 days, after this period $1 \mathrm{cc}$ of blood was taken and they were also sacrificed. Lastly, group 5 was determined as the subcutaneous simvastatin group. In this group, the SCI procedure was performed, subcutaneous simvastatin $(10 \mathrm{mg} / \mathrm{kg})$ from the first day (at 1sthour) was given to the rats every day until the3rd day. On the 4th day, $1 \mathrm{cc}$ of blood was taken and rats were sacrificed. In all the groups, before performing SCI, we obtained $1 \mathrm{cc}$ of blood from all the rats in order to assess biochemical alterations before the surgery and after treatment period.

\section{Histopathological examination}

All spinal cord samples were perfused with $200 \mathrm{ml}$ of $4 \%$ formaldehyde in a $0.1 \mathrm{M}$ phosphate-buffer for histological and immunohistochemical analysis. Formalin-fixed spinal cord sample sections $(5 \mu \mathrm{m})$ were stained with haematoxylin-eosin Nissl staining. All sections were irrigated in PBS and images were taken with an Olympus C-5050 digital camera mounted on an Olympus BX51 microscope. We assessed the spinal cord sections for oedema,

Tab. 1. Inflammation, haemorrhage and oedema categories of groups.

\begin{tabular}{|c|c|c|c|c|c|c|c|}
\hline & & $\begin{array}{l}\text { Control } \\
\text { group }\end{array}$ & $\begin{array}{l}\text { Sham } \\
\text { group }\end{array}$ & $\begin{array}{c}10 \mathrm{mg} / \mathrm{kg} \text { oral } \\
\text { simvastatin group }\end{array}$ & $\begin{array}{c}10 \mathrm{mg} / \mathrm{kg} \text { intraperitoneal } \\
\text { simvastatin group }\end{array}$ & $\begin{array}{c}10 \mathrm{mg} / \mathrm{kg} \text { subcutaneous } \\
\text { simvastatin group }\end{array}$ & $\mathrm{p}$ \\
\hline \multirow{3}{*}{$\begin{array}{l}\text { Inflammation } \\
\text { category }\end{array}$} & Absent (n) & 6 & 4 & 4 & 2 & 0 & \multirow{3}{*}{0.038} \\
\hline & Moderate (n) & 0 & 2 & 2 & 4 & 5 & \\
\hline & Severe $(n)$ & 0 & 0 & 0 & 0 & 1 & \\
\hline \multirow{3}{*}{$\begin{array}{l}\text { Haemorrhage } \\
\text { category }\end{array}$} & Absent (n) & 6 & 1 & 4 & 3 & 2 & \multirow{3}{*}{0.224} \\
\hline & Moderate (n) & 0 & 1 & 1 & 1 & 1 & \\
\hline & Severe $(n)$ & 0 & 4 & 1 & 2 & 3 & \\
\hline \multirow{3}{*}{$\begin{array}{l}\text { Oedema } \\
\text { category }\end{array}$} & Absent (n) & 6 & 0 & 2 & 1 & 0 & \multirow{3}{*}{0.0001} \\
\hline & Moderate (n) & 0 & 2 & 4 & 5 & 4 & \\
\hline & Severe $(n)$ & 0 & 4 & 0 & 0 & 2 & \\
\hline
\end{tabular}




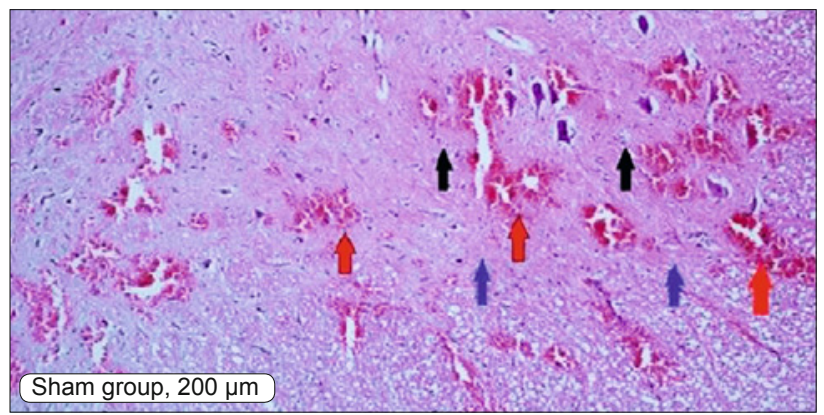

Fig. 1. Spinal cord section from Sham operated group. (x 100 magnification). (Red arrow; haemorrhage, blue arrow; oedema, black arrow; inflammation).

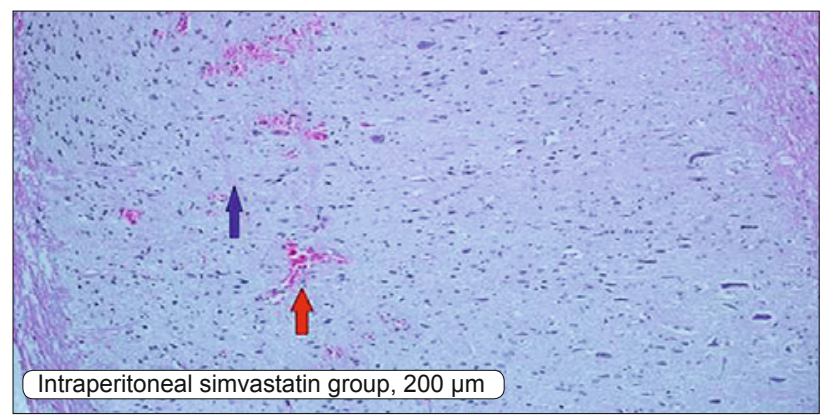

Fig. 2. Spinal cord section from $10 \mathrm{mg} / \mathrm{kg}$ intraperitoneal simvastatin group (x100 magnification). (Red arrow; haemorrhage, blue arrow; oedema, black arrow; inflammation).

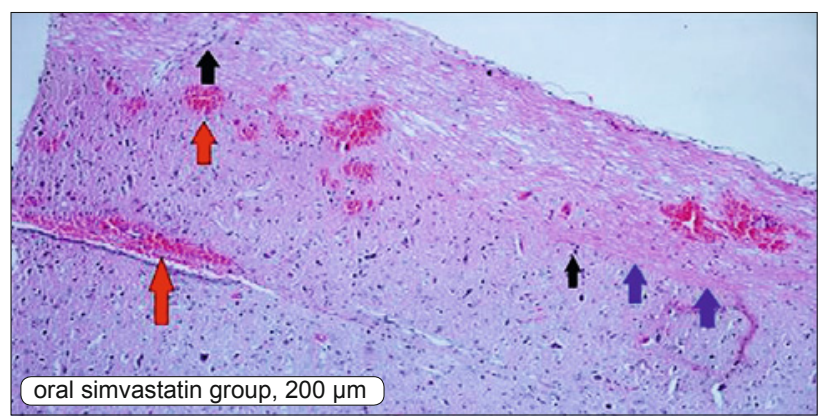

Fig. 3. Spinal cord section from $10 \mathrm{mg} / \mathrm{kg}$ oral simvastatin group (x100 magnification). (Red arrow; haemorrhage, blue arrow; oedema, black arrow; inflammation).

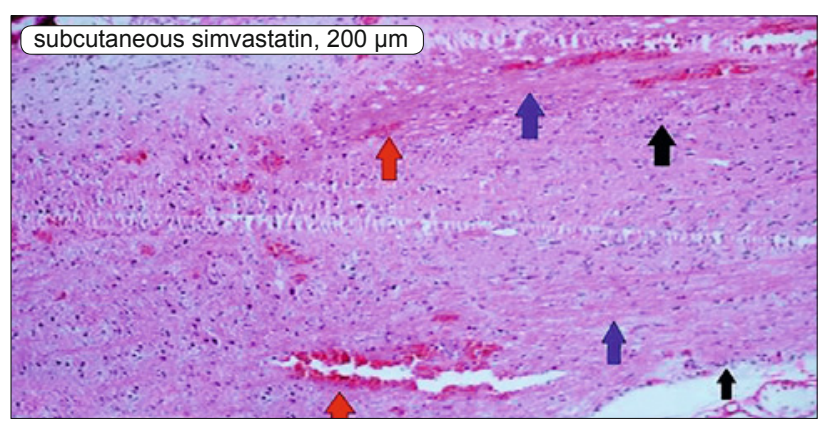

Fig. 4. Spinal cord section from $10 \mathrm{mg} / \mathrm{kg}$ subcutaneous group (x100 magnification). (Red arrow; haemorrhage, blue arrow; oedema, black arrow; inflammation). haemorrhage and inflammation under a microscope according to the system reported by Ducker et al. (18). Briefly, 0 means no pathological finding, while 1 means moderate and 2 means severe pathological alterations.

\section{Biochemical analyses}

All blood samples were taken before and after the SCI procedure with appropriate methods. Serum samples were obtained by centrifugation ( $3000 \mathrm{RPM}, 10 \mathrm{mins}$, at $4{ }^{\circ} \mathrm{C}$ ) and biochemical investigations were performed immediately. The concentrations of NSE and S100B were measured via commercial ELISA kits according to manufacturer recommendations (DRG, Germany).

\section{Statistical analysis}

The SPSS (Statistical Package for Social Sciences) version 15.0 for Windows program was used for all statistical analyses in this study. The Chi-Square test was used to compare qualitative data. The non-parametric Mann-Whitney U and Kruskal-Wallis tests were used to compare quantitative data, and post-hoc comparisons were performed via the least significant difference test. The results are presented as the mean \pm standard deviation for numeric values and as " $n$ " and "\%" for the qualitative values. Results with a $\mathrm{p}$ value lower than 0.05 were accepted to be statistically significant.

\section{Results}

The control group's oedema, haemorrhage and inflammation scores and S100 $\beta$ and NSE levels were significantly lower than the sham group and the three simvastatin treatment groups $(\mathrm{p}<$ 0.05 in all). When groups were compared in terms of oedema and inflammation histopathologically, the simvastatin group's scores were better than sham group $\left(x^{2}=29.77, p=0.0001\right.$ and $x^{2}=16.34$, $\mathrm{p}=0.038$ respectively). However, there were no significant differences between the treatment groups and the sham group in terms of haemorrhage scores $\left(x^{2}=10.6, p=0.224\right)$. When the 3 treatment groups (oral, intraperitoneal and subcutaneous simvastatin) were compared with each other in terms of inflammation, haemorrhage and oedema, there were no significant differences between the groups $\left(x^{2}=7.23, p=0.112 ; x^{2}=1.16, p=0.797\right.$ and $x^{2}=6.15, p$ $=0.188$; respectively) (Tab. 1, Figs $1,2,3,4$ ).

The ANOVA test showed that there were significant differences between groups in terms of NSE and S100B levels ( $\mathrm{F}=$ $2.9, \mathrm{p}=0,039$ and $\mathrm{F}=5.06, \mathrm{p}=0,004$ respectively). NSE and $\mathrm{S} 100 \mathrm{~B}$ levels were significantly higher in the sham group than in the control group $(\mathrm{p}=0.0017)$, while NSE and S100B levels were significantly lower in the treatment groups than the sham group. On the other hand, NSE and S100B levels were similar in all three treatment groups $(\mathrm{F}=2.74, \mathrm{p}=0,097, \mathrm{~F}=7.6, \mathrm{p}=0,125)$ (Fig. 5).

\section{Discussion}

The major finding of our study was that, simvastatin had significant neuroprotective effects in a rat SCI model. S100B and NSE levels, which are important and reliable indicators of 


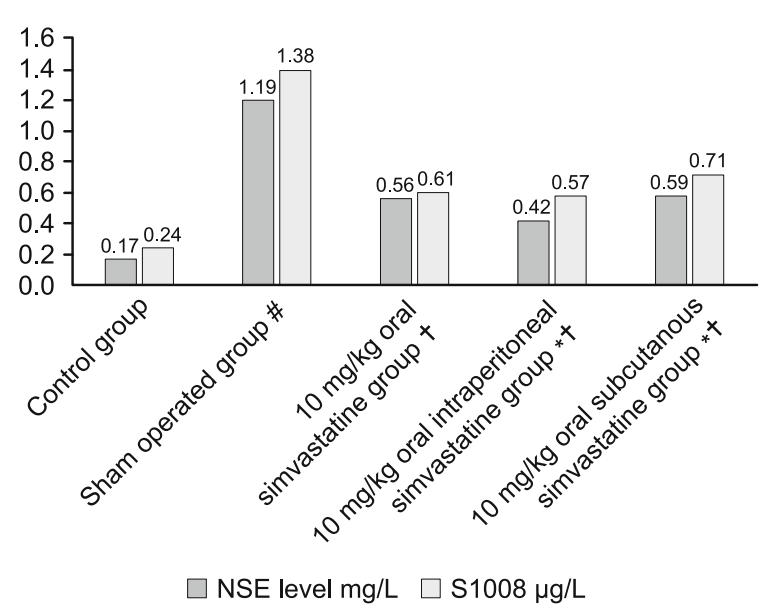

Fig. 5. NSE and S100B levels were significantly higher in sham group than control group $(p=0.0017)$. NSE and S100B levels were significantly different from sham group $(p=0,039$ and $p=0.004$ respectively); NSE and S100B levels were similar in all three treatment groups $(p=$ 0.097 and $p=0.125$, respectively).

neuronal damage, were significantly lower in the three treatment groups compared to the sham group. Besides, the histopathological findings of our study supported these results, as shown by the levels of oedema and inflammation. Finally, we found that oral, intraperitoneal and subcutaneous simvastatin showed similar positive impacts on SCI, indicating that neuroprotective effects are not associated with the route of administration.

Our results were consistent with previous literature reports regarding simvastatin. In the recently published study, Liang et al showed that $10 \mathrm{mg} / \mathrm{kg}$ of simvastatin could improve neurological function and regulate endothelial inflammatory response by reducing adhesion molecule-1 (ICAM-1) after a spinal cord injury in rats (19). In other studies, $10 \mathrm{mg} / \mathrm{kg}$ simvastatin was also shown to have ameliorative effects on SCI through different mechanisms; for instance, Gao et al reported that while simvastatin significantly increased the expression of brain-derived neurotrophic factor (BDNF) and glial cell line-derived neurotrophic factor (GDNF), it decreased expression of chondroitin sulfate proteoglycan (CSPG) and caspase-3 protein. Furthermore, it preserved the function of motor neurons (20). Also, Sohn et al created an Ischemia-reperfusion injury model from the tissue obtained from Sprague-Dawley rat foetuses and administered simvastatin. The authors found that oxygen and glucose deprivation (OGD) increased LDH release up to $214 \%$; however, simvastatin treatment attenuated cytotoxicity and also reduced the morphological alterations observed in motor neurons after OGD (21).

It can be seen from the above mentioned studies that simvastatin demonstrates biochemical, histopathological and clinical improvement in different experimental SCI models through different mechanisms. Our study added to these studies by assessing NSE and S100B levels as the measures of neuronal damage, which showed that simvastatin reduced the levels of NSE and S100B; thus providing a biochemical evidence of neuroprotective properties.
Our biochemical results were also supported by histopathological findings. Additionally, another aim of our study was to evaluate whether there were any differences in the terms of the administration route of simvastatin (oral, intraperitoneal and subcutaneous). Our results showed that the neuroprotective effects were similar in all 3 groups. To our knowledge, this is the first study, which demonstrates the positive effects of simvastatin as measured by NSE and S100B concentration.

High S100B levels induce neuro-inflammation via elevation of cytokines such as IL-6 and interferon gamma and S100B also activates apoptosis by the elevation of various apoptotic genes including c-fos, c-jun, bax, bel-x, p15, p21 (23); thereby causing neuron loss during injury. NSE is a $78 \mathrm{kDa}$ glycolytic enzyme involved in glucose metabolism and is mainly found in neuronal and neuroendocrine cells. The role of NSE in acute spinal cord injury (SCI) via various molecular mechanisms and pathways have been reported previously (24).

In parallel with the above- mentioned data, there were also some clinical studies, which showed that NSE and S100B levels could be used as a prognostic biomarker in SCI. In the clinical study by Du et al, serum levels of NSE and S100B protein were found to reflect the severity of spinal cord injury, and therefore, could be potential biomarkers in patients with an acute spinal cord injury (25). In another similar study, Wolf et al determined a significant correlation between serum S100B levels and vertebral spine fractures and also a significant correlation between serum S100B levels and SCI with neurologic deficit; however, unlike Du et al they did not find any association between NSE and SCI (25). Consistent with the findings of Wolf and colleagues, Marquardt et al, in their study consisting of rats with an experimental SCI model, reported that $\mathrm{S} 100 \mathrm{~B}$ was a reliable marker that enabled the prediction of SCI outcome, while NSE did not have this property (26). Although we did not evaluate any relationship between SCI severity and the levels of NSE and S100B, our results were similar to those mentioned studies in terms of increased NSE and S100B levels in SCI.

This study had certain limitations. Firstly, we could not evaluate the clinical severity and outcomes of rats because of technical insufficiencies. Another important limitation was the semiquantitative nature of our histopathological assessment, which was performed according to the prior study. Future studies may benefit from utilizing quantitative methods in order to determine histopathological alterations more accurately, such as: methods involving neuron or astrocyte cell counts.

\section{Conclusion}

In the current study, simvastatin $10 \mathrm{mg} / \mathrm{kg}$ (regardless of administration route) was found to have a significant positive impact on SCI in rats, as measured by histopathological and biochemical methods; however, although various studies were performed on this topic, the primary mechanism by which simvastatin demonstrates this effect is unknown. In future, detailed experimental or clinical studies, which evaluate other biomarkers might be crucial in understanding these mechanisms. 


\section{References}

1. Karacan I, Koyuncu H, Pekel Ö et al. Traumatic spinal cord injuries in Turkey: A nation-wide epidemiological study. Spinal Cord 2000; 38 (11): 697-701. DOI: 10.1038/sj.sc.3101064.

2. Kulbatski I, Mothe AJ, Nomura H, Tator CH. Endogenous and exogenous CNS derived stem/progenitor cell approaches for neurotrauma. Curr Drug Targets 2005; 6 (1): 111-126. DOI: 10.2174/1389450053345037.

3. Déry MA, Rousseau G, Benderdour M, Beaumont E. Atorvastatin prevents early apoptosis after thoracic spinal cord contusion injury and promotes locomotion recovery. Neurosci Lett 2009; 453 (1): 73-76. DOI: 10.1016/j.neulet.2009.01.062.

4. Hayashi M, Ueyama T, Nemoto K, Tamaki T, Senba E. Sequential mRNA expression for immediate early genes, cytokines, and neurotrophins in spinal cord injury. J Neurotrauma 2000; 17 (3): 203-218. DOI: 10.1089/neu.2000.17.203.

5. Stüve O, Youssef S, Steinman L, Zamvil SS. Statins as potential therapeutic agents in neuroinflammatory disorders. Curr Opinion Neurol 2003; 16 (3): 393-401. DOI: 10.1097/01.wco.0000073942.19076.d1.

6. Hayashi M, Ueyama T, Nemoto K, Tamaki T, Senba E. HMG CoA reductase inhibitors reduce ischemic brain injury of Wistar rats through decreasing oxidative stress on neurons. Brain Res 2005; 1037 (1-2): 52-58. DOI: 10.1016/j.brainres.2004.12.051.

7. Vaughan CJ, Delanty N. Neuroprotective properties of statins in cerebral ischemia and stroke. Stroke 1999; 30 (9): 1969-1973. DOI: 10.1161/01.str.30.9.1969.

8. Youssef S, Stüve O, Patarroyo JC et al. The HMG-CoA reductase inhibitor, atorvastatin, promotes a Th2 bias and reverses paralysis in central nervous system autoimmune disease. Nature 2002; 420 (6911): 78-84. DOI: 10.1038 /nature 01158 .

9. Saheki A, Terasaki T, Tamai I, Tsuji A. In Vivo and in Vitro BloodBrain Barrier Transport of 3-Hydroxy-3-Methylglutaryl Coenzyme A (HMG-CoA) Reductase Inhibitors. Pharm Res 1994; 11 (2): 305-311. DOI: 10.1023/A:1018975928974.

10. Zacco A, Togo J, Spence K et al. 3-hydroxy-3-methylglutaryl coenzyme A reductase inhibitors protect cortical neurons from excitotoxicity. J Neurosci 200323 (35): 11104-1111.

11. Asano T, Mori T, Shimoda T et al. Arundic acid (ONO-2506) ameliorates delayed ischemic brain damage by preventing astrocytic overproduction of S100B. Curr Drug Targets: CNS Neurol Dis 2005; 4 (2): 127-142. DOI: $10.2174 / 1568007053544084$.

12. Mrak RE, Griffin WST. The role of activated astrocytes and of the neurotrophic cytokine S100B in the pathogenesis of Alzheimer's disease. Neurobiol Aging 2001; 22 (6): 915-922. DOI: 10.1016/S0197-4580 (01)00293-7.

13. Hardemark HG, Ericsson N, Kotwica Z et al. S-100 protein and neuron-specific enolase in CSF after experimental traumatic or focal ischemic brain damage. J Neurosurg 1989; 71 (5 I): 727-731. DOI: 10.3171/ jns.1989.71.5.0727.
14. Anand N, Stead LG. Neuron-specific enolase as a marker for acute ischemic stroke: A systematic review. Cerebrovasc Dis 2005; 20 (4): $213-$ 219. DOI: $10.1159 / 000087701$.

15. Bharosay A, Bharosay VV, Varma M, Saxena K, Sodani A, Saxena R. Correlation of brain biomarker neuron specific enolase (NSE) with degree of disability and neurological worsening in cerebrovascular stroke. Indian J Clin Biochem 2012; 27 (2): 186-190. DOI: 10.1007/ s12291-011-0172-9.

16. Cayli SR, Kocak A, Yilmaz U et al. Effect of combined treatment with melatonin and methylprednisolone on neurological recovery after experimental spinal cord injury. Eur Spine J 2004; 13 (8): 724-732. DOI: 10.1007/s00586-003-0550-y.

17. Albayrak S, Atci IB, Kalayci M et al. Effect of carnosine, methylprednisolone and their combined application on irisin levels in the plasma and brain of rats with acute spinal cord injury. Neuropeptides 2015; 52:47-54. DOI: 10.1016/j.npep.2015.06.004.

18. Ducker TB, Kindt GW, Kempf LG. Pathological findings in acute experimental spinal cord trauma. J Neurosurg 1971; 35 (6): 700-708. DOI: 10.3171/jns.1971.35.6.0700.

19. Liang CL, Chen HJ, Liliang PC et al. Simvastatin and simvastatinEzetimibe improve the neurological function and attenuate the endothelial inflammatory response after spinal cord injury in rat. Ann Clin Labor Sci 2019; 49 (1): 105-111.

20. Gao K, Wang G, Wang $Y$ et al. Neuroprotective Effect of Simvastatin via Inducing the Autophagy on Spinal Cord Injury in the Rat Model. BioMed Res Internat 2015; 2015. DOI: 10.1155/2015/260161.

21. Sohn HM, Hwang JY, Ryu JH et al. Simvastatin protects ischemic spinal cord injury from cell death and cytotoxicity through decreasing oxidative stress: In vitro primary cultured rat spinal cord model under oxygen and glucose deprivation-reoxygenation conditions. J Orthop Surg Res 2017; 12 (1). DOI: 10.1186/s13018-017-0536-9.

22. Haque A, Polcyn R, Matzelle D, Banik NL. New insights into the role of neuron-specific enolase in neuro-inflammation, neurodegeneration, and neuroprotection. Brain Sci 2018; 8 (2). DOI: 10.3390/brainsci8020033.

23. Zimmer DB,Weber DJ. The calcium-dependent interaction of S100B with its protein targets. Cardiovasc Psychiat Neurol 2010. DOI: 10.1155/2010/728052.

24. Du W, Li H, Sun J et al. The prognostic value of serum neuron specific enolase (NSE) and s100b level in patients of acute spinal cord injury. Med Sci Monitor 2018; 24:4510-4515. DOI: 10.12659/MSM.907406.

25. Wolf H, Krall C, Pajenda G et al. Alterations of the biomarker S-100B and NSE in patients with acute vertebral spine fractures. Spine J 2014; 14 (12): 2918-2922. DOI: 10.1016/j.spinee.2014.04.027.

26. Marquardt G, Setzer M, Theisen A, Tews DS, Seifert V. Experimental subacute spinal cord compression: Correlation of serial S100B and NSE serum measurements, histopathological changes, and outcome. Neurol Res 2011; 33 (4): 421-426. DOI: 10.1179/1743132810Y.0000000005.

Received May 19, 2020. Accepted May 23, 2020. 